\title{
TASAS DE DEPENDENCIA Y TASAS DE AHORRO AGREGADO: CORRECCIONES Y ANÁLISIS DETALLADO
}

\author{
RICHARD E. BILSBORROW* \\ Universidad de Carolina del Norte
}

EN EL DECENIo pasado se desarrolló abundante discusión teórica acerca de los efectos negativos que en los países menos desarrollados tiene el crecimiento de la población sobre el ahorro, la inversión, la productividad del trabajo y, en general, sobre el crecimiento económico. Sin embargo, el desarrollo de trabajos empíricos ha sido más bien limitado. ${ }^{1}$

El artículo de Nathaniel Leff que se publicó en la American Economic Review y en Demografia y Economía [25] fue el primer trabajo que aporta evidencia estadística acerca de los efectos que el crecimiento de la población puede tener en las tasas de ahorro de los países en desarro11o. Posteriormente, Leff al analizar el caso de América Latina en una comunicación a esta revista [25a] amplió su análisis de las relaciones entre crecimiento de la población y ahorro.

El autor del artículo presente tiene serias dudas sobre la validez de los diversos resultados a los que Leff llega. El propósito de este comentario es discutir los siguientes aspectos: 1) mostrar que los resultados obtenidos (por Leff) son en parte producto de un error en la escala de

* Departamento de Bioestadística, Universidad de Carolina del Norte en Chapel Hill, Carolina del Norte, U.S.A. Esta investigación se realizó cuando el autor desempeñaba el cargo de profesor asistente de economía en la Universidad de Nueva York. El autor agradece los valiosos comentarios de Peter Albin, Robert P. Althauser, Ansley J. Coale, Charles R. Frank, Jr., Thomas Frejka, Allen Kelley y Lister Taylor. El financiamiento para esta investigación se obtuvo del Consejo de Población. El Centro de Investigación sobre Desarrollo Económico de la Universidad de Michigan proporcionó diversas facilidades. En particular agradezco a Nathaniel Leff el haberme proporcionado una copia de sus datos, así como a Alfredo Gutiérrez por su ayuda como asistente. Ninguna de las Instituciones o personas antes mencionadas tienen responsabilidad por el contenido de este trabajo.

Las referencias de las páginas de esta versión corresponden a Demografía Y Economía. El autor tiene la versión en inglés publicada en la American Economic Review (copias del mismo pueden solicitarse al autor).

1 La mayor parte de la investigación empírica se ha enfocado al análisis de los efectos en la dirección opuesta, es decir, del desarrollo económico sobre el crecimiento de la población. Ver Adelman [3], Drakatos [9], Krishnamurty [20], Phillips et al. [31], y numerosas referencias en la literatura demográfica, en particular sobre los países desarrollados. 
medición en una de las variables independientes; y 2) indicar algunos problemas conceptuales y metodológicos que van más allá de los ya expuestos en un comentario anterior sobre el artículo de Leff (American Economic Review, junio de 1971) los cuales ponen en entredicho el procedimiento seguido en relación con la selección de una muestra más grande de países en general y de América Latina en particular.

Es importante destacar aquí las limitaciones de los resultados de Leff," básicamente por ser un trabajo que se cita con bastante frecuencia en la literatura especializada sobre economía y demografía. ${ }^{3}$

\section{El ERROR EN LA ESCALA DE MEDICIÓN Y SUS IMPLICACIONES}

En su artículo basado en un análisis transversal con observaciones para 74 países, 47 de los cuales los clasifica como subdesarrollados, Leff investiga los efectos separados del ingreso y del crecimiento de la población (medido a través de la relación de dependencia) sobre el ahorro.

Los principales resultados de la regresión (ecuación 1, p. 84 y ecuación 1 en el cuadro 4, p. 86) se reproducen en el cuadro 1, ecuaciones 1 y 3 . Las variables utilizadas son las siguientes (en el artículo de Leff aparecen mayores detalles):

$$
\begin{aligned}
S= & \text { Ahorro interno bruto } \\
Y: & \text { Producto interno bruto } \\
N= & \text { Población } \\
D_{1}= & \text { Proporción de población menor de } 15 \text { años respecto a la po- } \\
& \text { blación de } 15-64 \\
D_{2}= & \text { Proporción de población mayor de } 64 \text { años con respecto a la } \\
& \text { población de } 15-64 \\
g= & \text { Tasa de crecimiento como porciento del ingreso real per ca- } \\
& \text { pita. }
\end{aligned}
$$

2 En dos recientes y muy leídos artículos de la literatura económico-demográfica, se encuentra lo siguiente: Leff, en un análisis empírico reciente, sugiere que una relación directa entre la tasa de dependencia y la tasa de ahorros es al menos tan significativa como la relación indirecta vía ingreso per capita (E. Hoover, $[14]$, p. 68) "El mejor estudio empírico sobre las relaciones entre ingreso y tamaño de la familia fundamenta en forma moderada el supuesto de Carle-HooverDemeny" (Robinson y Horlacher, [33], pie de página 68). Por último, los resultados de las regresiones de Leff, sin ser criticadas, se han incorporado al menos en una investigación ya terminada sobre un modelo económico-demográfico de simulación [4].

3 Esta nota se centrará en los resultados de Leff para países en desarrollo ya que las implicaciones de política son para estos países, en potencia más importantes (dicho por Leff, c.f. pp. 90-91). También se enfocará en la variable dependiente $S / Y$ que representa la tasa de ahorro, más que en $S / N$, ahorros por persona, por ser este último de poco interés teórico y adolecen de limitaciones metodológicas (Véase el apéndice B). 
Cuadro 1

CORRECCIÓN DE LOS RESULTADOS EMPíRICOS DE LEFF PARA LAS REGRESIONES $\ln S / Y$

(los valores de $\mathrm{t}$ aparecen entre paréntesis)

\begin{tabular}{|c|c|c|c|c|c|c|}
\hline \multirow{2}{*}{ Ecuacion } & \multicolumn{6}{|c|}{ Variables independientes } \\
\hline & $\ln \mathbf{Y} / \mathbf{N}$ & in $D_{1}$ & $\ln D_{2}$ & $\ln 8$ & $\overline{\mathrm{R}}^{2}$ & $\mathbf{F}$ \\
\hline \multicolumn{7}{|l|}{ Paisas en desarrollo } \\
\hline $\begin{array}{l}\text { (1) Resul tados origina- } \\
\text { les de Ieffb }\end{array}$ & $\begin{array}{l}0.129 / 1 \\
(1.849)\end{array}$ & $\begin{array}{l}-1.230 \mathrm{~d} / \\
(2.764)\end{array}$ & $\overrightarrow{\left(2.446^{e}\right.}$ & $\stackrel{0.023^{\mathrm{d}} /}{(2.808)}$ & 0.242 & $4.668^{\mathrm{a}}$ \\
\hline $\begin{array}{l}\text { (2) Ecuacion de Leff, } \\
\text { corregida }\end{array}$ & $\begin{array}{l}0.130 \% \\
(1.785)\end{array}$ & $\begin{array}{l}-1.227^{\circ} \\
(2.491)\end{array}$ & $\begin{array}{l}-0.480 \mathrm{~d} \\
(2.036)\end{array}$ & $\begin{array}{l}0.579 \\
(0.742)\end{array}$ & 0.131 & $2.586^{\mathrm{c}} f$ \\
\hline \multicolumn{7}{|l|}{ Todos Ios patises } \\
\hline $\begin{array}{l}\text { (3) Resultados orifina- } \\
\text { les de Leff } y^{\text {na }}\end{array}$ & $(2.879)$ & $(4.641)$ & (2.562) & $(3.279)$ & 0.570 & 25.160 d \\
\hline $\begin{array}{l}\text { (4) Ecuacion de Leff, } \\
\text { oorreEida }\end{array}$ & $\frac{0.16)^{\mathrm{d}} /}{(2.908)}$ & $\frac{-1.326^{d / d}}{(4.506)}$ & $\frac{-0.422^{d / d}}{(2.713)}$ & $\begin{array}{l}0.077 \\
(1.229)\end{array}$ & 0.526 & $20.106^{d /}$ \\
\hline
\end{tabular}

a Regresiones doble-logarítmicas incluido el término constante.

- Para 47 países en desarrollo. La ecuación 2 se estimó para 43 países. Se eliminaron República Dominicana, Ghana, Marruecos y Uruguay, por registrar una tasa de crecimiento del ingreso per capita igual a 0 en el período 1960-1964, ya que el $\ln g$ no existe para $\mathrm{g}=0$.

c Significativa al nivel 0.1 .

¿ Significativa al nivel 0.01 .

c Leff en este caso, en forma equivocada, lo considera como significativo al nivel de 0.01 .

f Para 74 países. La ecuación 4 se estimó con base en la información para 70 países (véase nota $b$ ).

Su conclusión más importante es que a una mayor tasa de dependencia, producto de una mayor población joven y senil, correlativamente tiende a disminuir la tasa de ahorro de manera sistemática; dicha relación inversa se verifica en todos los países, incluidos los menos desarrollados (pp. 82, 83 y passim).

Leff utiliza una transformación doble logarítmica con objeto de evitar el problema de heterocedasticidad que ocurre con frecuencia en las regresiones basadas en datos transversales. La transformación logarítmica es un recurso válido sólo cuando se tienen valores positivos distintos de cero. En el caso que nos ocupa, la transformación no se justifica ya que existen cuatro valores de la variable $g$ que son iguales a cero. Para no eliminar estos cuatro países (todos ellos subdesarrollados; véase la nota $b$ del cuadro 1 . Leff asigna como valor de $g$ a estos cuatro países un número positivo muy pequeño: $1 \times 10^{-9}$. El logaritmo natural para $1 \times 10^{-9}$ es -18.4207 , para los otros 43 países subdesarrollados el lo- 
garitmo natural varía entre $-0.9163 \mathrm{y}+2.1861 .{ }^{4}$ De esta manera, con el procedimiento de Leff se derivan cuatro valores extremos, los que modifican por completo los resultados empíricos. Al eliminar los cuatro países se observa que: 1) la variable $g$ no es estadísticamente significativa; ${ }^{5}$ 2) la variable dependencia $D_{1}$ reduce su nivel de significación; y 3) la significación estadística de la ecuación (con base en el estadístico $F$ ) se reduce notablemente. Con base en lo anterior, hay serias reservas para que la nueva ecuación -2) en el cuadro 1- se acepte en forma generalizada para apoyar su hipótesis. Es importante señalar que el error de medición está presente también en otras ecuaciones utilizadas por Leff y lo mismo ocurre con las ecuaciones que presentan Adams [2] y Gupta [12] en sus comentarios al artículo de Leff. Una corrección a los resultados de Leff para $\ln S / Y$ se presenta en el cuadro 1 -ecuación 4de este trabajo. ${ }^{6}$

\section{El PROBlema dE LA HETEROgeneidAd DE LA MUESTRA}

Otro problema relacionado con el anterior es el de la heterogeneidad de la muestra. En el análisis transversal de los llamados países menos desarrollados existe gran heterogeneidad, problema que no queda resuelto por el solo hecho de "controlar" el ingreso per capita al incluir esta variable en la ecuación. Si se toman como punto de partida elementos tanto conceptuales como estadísticos, se discutirá en primer lugar el pro-

4 Los valores de $g$ fueron tomados por Leff como tasas porcentuales de crecimiento y sus valores numéricos van de 0.4 a 7.3 (para países en desarrollo) o a 8.9 (cuando se incluyen todos los países), sus valores correspondientes al logaritmo natural se mencionan en el texto (tanto los datos originales como las transformaciones logarítmicas correspondientes aparecen en el listado de datos de la computadora elaborados por Leff). La asignación arbitraria hecha por Leff de valores $1 \times 10^{-9}$ para cuatro países, se considera como un error de medición. Aun cuando añadir un número tan pequeño a cualquiera 0 todos los datos en la muestra no produce ningún efecto de importancia, ni en su tamaño ni en el logaritmo, como tampoco en cualquier otra forma no logarítmica. Es por ello que el error puede ser o bien de medición o debido a la asignación arbitraria de un valor a una variable. En este último caso el valor asignado es absurdo e influye en los resultados, como se muestra en el texto.

5 Esto es sorprendente ya que se esperaría que la tasa de crecimiento del ingreso per capita estuviese correlacionada con la tasa de ahorro, independientemente de la dirección de causalidad. Dicha hipótesis parecería tener respaldo en la teoría de Fredman acerca de la función consumo [10], en la que la propensión marginal a consumir, correspondiente al ingreso "transitorio" (aumentos en), es menor que la propensión marginal a consumir que corresponde al ingreso "permanente", pero, de aceptarse esta hipótesis, ésta se aplicaría al análisis de series de tiempo. Evidencias opuestas a las que aquí se presentan - para muestras más pequeñas en número y que incluyen países desarrollados y en desarrollo- se encuentran en Houthakker [15] y Modigliani [29].

6 Correcciones a las otras ecuaciones de Leff (con $S / N$ como la variable de. pendiente) pueden solicitarse al autor. 
blema de los "puntos extremos", y luego se presentarán evidencias que indican que la variancia obtenida después de hacer la corrección de los resultados empíricos, por el error de medición, se explica en gran medida por la presencia de puntos extremos.

En la muestra de Leff, ciertos países en desarrollo no pertenecen al mismo nivel o clase de la mayoría (más representativos) de estos países, de acuerdo con algunos indicadores socioeconómicos o demográficos. Este es el caso de Grecia, Israel, Puerto Rico y España y en menor medida de Argentina, Chipre, Malta, Portugal, Trinidad, Uruguay y Venezuela.

En la clasificación hecha por Naciones Unidas, o si se utiliza el índice compuesto de modernización elaborado por Harbinson, la mayoría de los economistas especialistas en desarrollo ${ }^{7}$ no consideran a los primeros cuatro países como subdesarrollados, por lo cual los resultados estadísticos que se derivan de la incorporación, hasta cierto punto dudosa, de ciertos países no puede considerarse que tengan relevancia general alguna para los países en desarrollo. ${ }^{8}$

Aún más, desde el punto de vista estadístico, se puede argumentar que estos cuatro países constituyen un buen ejemplo de "puntos extremos". Las razones de dependencia de Grecia y España están muy por debajo de los valores respectivos pertenecientes a los llamados de menor desarrollo, mientras que Israel y Puerto Rico tienen niveles de ingreso per capita mucho más elevado. ${ }^{9}$ En una gráfica de puntos se aprecian fá-

7 Muchas de las publicaciones de Naciones Unidas no concuerdan con su punto de vista. Por ejemplo, en la última publicación La situación económica mundial, 1969-70 [37], como en publicaciones previas, todos los países mencionados antes no se consideran como subdesarrollados, exceptuando tres países marginales de América Latina: Argentina, Uruguay y Venezuela. Una segunda clasificación de los países, mediante un sistema de correspondencia implica un ordenamiento similar [28]. Por último, tal vez el mejor intento de clasificación de países por su nivel de modernización es el de Harbinson [13], quien los clasifica con base en un "indice compuesto de desarrollo" (apéndice, pp. 97-99): Israel (18), Argentina (22), Puerto Rico (23), Uruguay (25), Venezuela (31), España (32), Grecia (34) y Portugal (46). Con fines comparativos: Japón (21), Italia (24), Polonia (26), Panamá (33), Trinidad (35) y los "verdaderos" países en desarrollo se encuentran entre los lugares 40 y 112 de la clasificación. Aún más, esta clasificación no incluye ni la variable dependencia ni otras variables demográficas, las que de haberse incluido aumentarían en forma considerable la posición de Grecia, España y Portugal vis a vis Venezuela, Panamá y los "verdaderos" países de escaso desarrollo. Por último, todos los países a que se hace referencia aquí y en el texto, fueron poblados en principio por poblaciones europeas, con la excepción de Venezuela y Trinidad.

$8 \mathrm{La}$ presente discusión, favorece a Gupta, op. cit., pero llega más lejos: primero, porque en apariencia no hace la corrección mencionada, por lo que el incluir los cuatro países que adolecen del error de medición (de los 16 países que conforman el grupo II, tres presentan la limitación mencionada) los resultados se distorsionan. En segundo lugar, su grupo III carece de significado por la mezcla de países que hace, que incluye desarrollados (Polonia y Japón), semidesarrollados y verdaderos países de menor desarrollo (véase también a Horbinson, [13]). Incluso, estos países no son los mismos que Leff consideró como subdesarrollados.

9 La justificación estadística para considerar los cuatro países como puntos ex- 


\section{Gráfica 1}

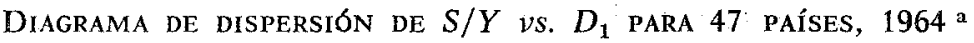
$S / Y$

$\left[\begin{array}{c}25 \\ 20 \\ \substack{\text { Grecio } \\ 0} \\ - \\ 34\end{array}\right.$
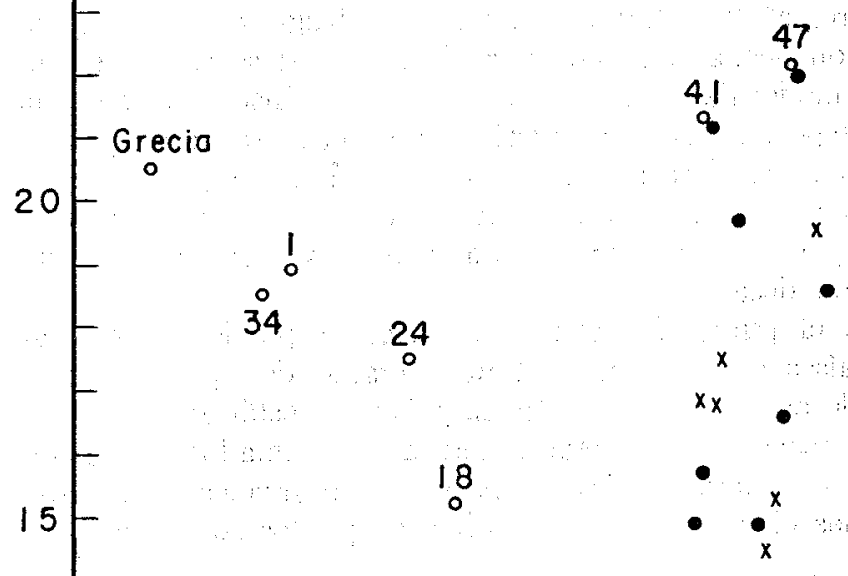

18

- $e_{\mathrm{x}}^{\mathrm{x}}$

46
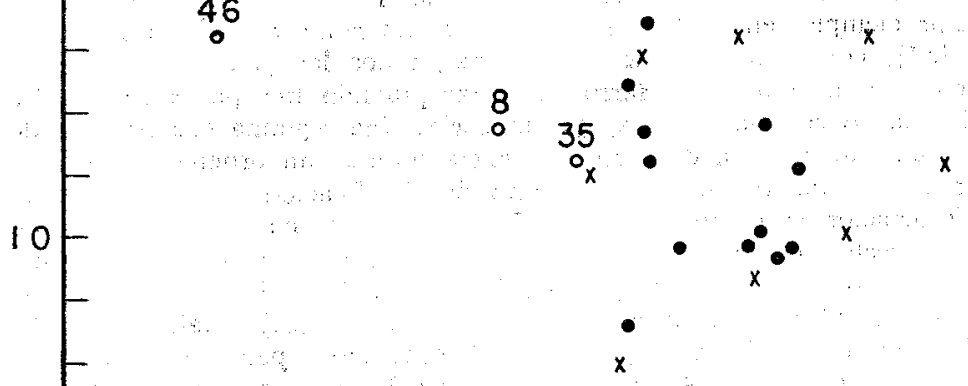

- Paises Marginales

- Países de Africa y Asia

x Paises de América Latina

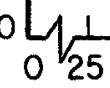

30

35

40

45

50

0 ,

a Obsérvese la localización relativa de los países marginales de escaso desarrollo. Se identifican con claridad los dos extremos con respecto a $D_{1}$. Los otros países marginales de escaso desarrollo son: (1) Argentina; (8) Chipre; (18) Israel; (24) Malta; (34) Portugal; (35) Puerto Rico; (41) Trinidad; (46) Uruguay; y (47) Venezuela. 
cilmente estos dos aspectos (véanse, por ejemplo, en la gráfica 1 los valores respectivos de $S / Y$ y $D_{1}$ ).

La dependencia en los resultados de Leff frente a la presencia de puntos extremos se presenta empíricamente en el cuadro 2. Al confrontar los resultados originales (ecuación 2 en el cuadro 1) se puede apreciar que tanto en el esquema conceptual como en los datos que maneja Leff, la relación entre tasa de dependencia y tasa de ahorro es prácticamente inexistente en los países en desarrollo (véase la sección III)..$^{10}$

El problema de la heterogeneidad que es de gran importancia en los

tremos se deriva de distintos enfoques. Todos parten del supuesto de que los valores de $D_{1}$ y $Y / N$ están normalmente distribuidos, supuesto necesario en las pruebas estadísticas relativas a muestras pequeñas y el cual también se requiere en el análisis de regresión. Estos enfoques incluyen: a) Hacer estimaciones de máxima verosimilitud de las medias y desviaciones estándar de $D_{1}$ y $Y / N$, cuyos valores son $41.655,5.844,314.74$ y 251.87 , respectivamente. En el caso de Grecia el valor de $D_{1}$ tiene desviaciones estándar de más de 2.58 por debajo de la media, por lo cual Grecia es diferente del resto de los países, con un nivel de significación de 1\%; lo mísmo se aplica a España, en cuyo caso el nivel de significación es de $2 \%$; los valores de $Y / N$ de Israel y Puerto Rico están a más de dos desviaciones estándar por arriba de la media, por lo que en forma similar se consideran como puntos extremos; b) en 1950 Gubbs [11] sugirió una técnica para determinar en qué condiciones dos observaciones muy grandes o muy pequeñas pertenecientes a una población normalmente distribuida, resultaban estadísticamente puntos extremos. Su técnica requiere cálcular la suma de cuadrados con respecto a la media de la muestra con y $\sin$ los dos puntos extremos y comparar la razón con algunos valores tabulados que él estimó. Al utilizar este procedimiento se llega a la misma conclusión que antes, los mismos pares de países resultan puntos extremos (para $D_{1}$ al nivel de significación del $5 \%$ y para $Y / N$ al $1 \%)$; c) Dixon y Massey ([8], pp. 275-276) proporcionaron un tercer procedimiento, el cuial da resultados similares (detalles de los tres procedimientos se pueden solicitar al autor).

Por el hecho de que las pruebas antes mencionadas parten del supuesto de que la población está normalmente distribuida, ninguno resulta tan "vigoroso" como uno quisiera. Además, aquí el enfoque es un examen univariado y lo que se recomienda es un examen bivariado del par de valores $\left(Y / N\right.$ y $\left.D_{1}\right)$ con el supuesto de normalidad bivariada. A este respecto, es importante subrayar que los cuatro países en cuestión registran valores moderadamente extremos en la otra variable del par mencionado, o sea, Grecia y España para los valores de $Y / N$ e Israel y Puerto Rico para $D_{1}$. Véase también la clasificación con base en el índice compuesto citado en la nota 7.

10 Leff intenta afrontar el problema de la heterogeneidad de la muestra en su réplica a Adams (American Economic Review, junio de 1971, p. 879). Su técnica, que consiste en incluir una variable dummy (un valor de uno para los 47 países llamados en desarrollo, y cero para los países más desarrollados) no resuelve el punto clave de la heterogeneidad interna de los países en desarrollo (tampoco de los países desarrollados). Por ejemplo, la ecuación de Leff (después de la corrección en la sección 1) para los 33 "verdaderos" países en desarrollo, es (el valor de $t$ aparece entre paréntesis):

$$
\begin{aligned}
& \ln S / Y=1.295+\underset{(1.208)}{0.121} \ln Y / N+\underset{(0.297)}{0.312 \ln } D_{1}-\underset{(-1.963)}{0.518 \ln } D_{2}+\underset{(1.290)}{0.16 \ln g} \text { g; } \\
& {\left[\mathrm{R}^{2}=0.154\right]}
\end{aligned}
$$


trabajos de tipo transversal, fue oportunamente discutido -Klein [19], pp. 212 ss.-. Este problema no sólo es privativo del trabajo de Leff, también aparece en la mayor parte de los trabajos recién publicados sobre países en desarrollo.

La falta de pruebas de sensibilidad sobre la presencia o ausencia de países marginales, explica que con frecuencia se obtengan regresiones aceptables que por otra parte carecen de significado para países en desarrollo. Así, los resultados que no pasan dichas pruebas no pueden considerarse de validez general.

\section{UN ENFOQUE ALTERNATIVO}

Una dificultad se presenta al estudiar los determinantes económicos y demográficos de las tasas de ahorro interno bruto en distintos países: la estructura de ahorros puede variar de modo considerable entre países debido al nivel de desarrollo, la estructura de la producción, la distribución del ingreso, factores institucionales relativos a impuestos y su administración, comercio exterior, etc. En la medida en que los factores demográficos inciden también sobre los componentes del ahorro y por lo tanto en su estructura, el efecto de un cambio demográfico dado tendrá un impacto distinto según sean las diferencias en la estructura de ahorros de los países. ${ }^{11} \mathrm{El}$ argumento de Leff parte del supuesto de que la variable "dependencia", es de importancia en cuanto tiene un efecto en los ahorros de la familia (p. 78): en familias numerosas el ahorro per capita es menor, así como también lo es el ahorro familiar. Mientras que el ahorro de las familias es una fuente importante de ahorros en los países desarrollados, en los cuales los ingresos familiares son más elevados, esto plantea la duda sobre si dicho modelo, basado en los ahorros familiares, es el paradigma adecuado para investigar el comportamiento del ahorro en los países de menor desarrollo.

Por otra parte, sólo es posible tener alguna idea de la importancia relativa de los ahorros familiares en 16 de los 47 países analizados por

Para los 10 países marginales el resultado es:

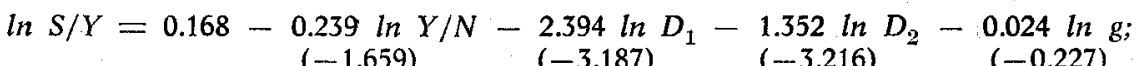

$$
\begin{aligned}
& {\left[\mathrm{R}^{2}=0.762\right]}
\end{aligned}
$$

En la primera ecuación, el signo de la variable $D_{1}$ es el opuesto al de la hipótesis, y en la segunda, el signo de $Y / N$ es distinto del esperado.

Es claro que estos conjuntos de países corresponden a universos socioeconómicos y demográficos muy distintos, por lo que la prueba de Chow [5] resulta innecesaria.

11 Es obvia la utilidad de contar con evidencias sobre los determinantes (económicos, demográficos, etc.) de los componentes del ahorro (hogares, empresas, gobierno y el exterior). El autor está realizando una investigación sobre el tema. 
Leff. ${ }^{12}$ Los ahorros familiares representaron la mitad o más del ahorro interno neto en sólo cinco de los 16 países ( $\mathrm{y}$ dos de los cinco - Grecia y Malta - no son considerados como países en desarrollo por Naciones Unidas, ni por este autor, véase la nota 7). Además, de los 15 países (serían 13 si se excluyen Grecia y Malta) donde es posible verificar esto, en seis -casi la mitad-, los ahorros del sector público son mayores que los privados (familiares y corporaciones privadas). Aquí de nuevo los aspectos institucionales del ahorro en los países en desarrollo (junto con las diferencias en el nivel de ingreso per capita) difieren con mucho de los de los países desarrollados, por lo cual la explicación de los efectos de la dependencia sobre los ahorros familiares resulta poco adecuada. ${ }^{13}$

Incluso los bajos valores de $R^{2}$ en las ecuaciones 2), 5) y 6) sugieren la necesidad de contar con un marco conceptual distinto a fin de investigar si las tasas de dependencia afectan las tasas de ahorro.

El marco teórico que aquí se propone, toma en consideración dos características de los países en desarrollo que varían de manera significativa entre ellos y las cuales pueden afectar las tasas de ahorro: la estructura de la producción y la importancia que tiene para sus economías el comercio internacional. En la mayoría de estos países, los productores del sector industrializado de la economía obtienen mayores utilidades brutas que los de la agricultura y los servicios. Dentro del sector industrial, las corporaciones dan cuenta en gran medida de estas utilidades. En la mayor parte de los países en desarrollo, los únicos sectores en donde predominan las corporaciones son la minería (incluido el petróleo) y las manufacturas. De aquí que países con el mismo nivel de ingreso per capita, pero en los cuales la minería y las manufacturas registran una elevada participación en el producto, las tasas de ahorro son por lo general más elevadas. Para tomar en cuenta esta característica se introduce la variable $O_{m m} / Y$ que representa la proporción del valor agregado de la minería y manufacturas. ${ }^{14}$

En forma similar, en los países en desarrollo el sector exportador es mucho más "comercializado" que el sector no exportador debido a que sus productos agrícolas y minerales de exportación son controlados por

12 Con datos de Naciones Unidas [38]. Las cifras corresponden a 1964, año que Leff utiliza (y no es atípico).

13 Los ahorros familiares medios representan alrededor del $33 \%$ de los ahorros en sólo dos países, y más del $40 \%$ en sólo uno de los 14 de América Latina para los que se obtuvieron datos referentes al período 1955-1967. Véase King [18], p. 6. Como regla, los ahorros del gobierno son los más importantes en los países africanos.

14 En tanto esta variable registra una alta correlación con $Y / N$, resulta ser una variable de "control" aceptable en el examen de los efectos de $D_{1}$ ya que incorpora no sólo los efectos del nivel de ingreso sino también la infulencia de la estructura del ingreso. El uso de $O_{m m} / Y$ como variable es un método alternativo que podría hacerse extensivo a todos los sectores a través de la estimación de propensiones marginales a ahorrar. En alguna medida esto es lo mismo que estimar propensiones marginales a ahorrar, con diferentes participaciones del ingreso, que es el enfoque clásico usual. 
corporaciones transnacionales y por los mismos gobiernos (por ejemplo federaciones de productores de café en América Latina y Consejos de Mercado en África). Para tomar en cuenta este aspecto se introduce la variable $X / Y$ (donde $X$ representa las exportaciones de bienes y servicios) como una medida de economía abierta. ${ }^{15} \mathrm{La}$ base teórica que justifica la utilización de estas dos variables es que los factores demográficos afectan el ahorro agregado a través de su efecto en los ahorros de los gobiernos y de las transnacionales más que en los ahorros familiares. ${ }^{16}$ Las variables $O_{m m} / Y$ y $X / Y$ se justifican también al reconocer el papel central que juegan tanto directa como indirectamente en la determinación de la base fiscal en las economías de los países de escaso desarrollo. ${ }^{17}$

Estas variables junto con $D_{1}$ y $D_{2}$ se utilizaron en las regresiones de $S / Y$. Los resultados se presentan en el cuadro 3. Al comparar las ecua-

15 Es posible que una justificación alternativa para $X / Y$ pueda ser igualmente factible: dada la existencia de control de permisos de importación en la mayoría de los países en desarrollo, el nivel de $X$ determina el nivel de $M$, el cual es, dada la importancia de las tarifas arancelarias de las importaciones como fuente de ingresos en estos países, un determinante importante de los ingresos del gobierno; y si se mantiene todo lo demás constante, de los ahorros gubernamentales. La elección de $X / Y$ en lugar de $M / Y$ o $(X+M) / Y$ se determinó a posteriori. Además, se ha hecho el hallazgo de que las exportaciones son un determinante importante de las tasas de ahorro. Landau [23], Cheney y Pekestein (4⿳) para países de América Latina y Lee [24].

16 Al afirmar Leff que "No hay una razón evidente de por qué la tasa de dependencia debería influir sobre los ahorros corporativos" (p. 78), se podría explicar el efecto de la dependencia en los ahorros : corporativos como sigue: a mayor crecimiento de la población mayores tasas de dependencia, mayor consumo de alimentos, etc. (y menor consumo de artículos manufacturados de "lujo"), lo que conduce a un menor volumen de ventas, de las utilidades y de ahorros netos de las corporaciones. En la medida en que las familias en los paises en desarrollo son "ahorradores-objetivo" puede darse la secuencia anterior, al mantener constantes los ahorros personales. Estudios sobre presupuestos familiares en los países en desarrollo (como también en los desarrollados) en parte fundamentan lo anterior. Por ejemplo Massell y Heyer. [27] observaron en una muestra de 300 familias de clase media de Nairobi, que en las familias grandes existe una tendencia a consumir más alimentos y menos de otros bienes. Véase también Kelly [17]. $\mathrm{Si}$ las corporaciones pagan impuestos más altos, estos ahorros potenciales por supuesto se transfieren parcialmente al sector público, pero esto de ningún modo afecta lo que aquí se discute, o sea la poca importancia de los ahorros familiares.

17 La administración fiscal en los países menos desarrollados está tan "subdesarrollada" como lo están sus economías. Esto explica que los gobiernos de los países en desarrollo recurran a los impuestos sobre ingresos, ventas y productos industriales de las corporaciones, como forma de obtener la mayor parte de los ingresos. Los primeros dos dependen en gran medida de $O_{m m}$ y el último del nivel de las importaciones, pero dado el racionamiento de permisos de importación, están afectados a su vez por el nivel de las exportaciones. El que dichos impuestos aumenten o disminuyan los ahorros nacionales dependerá de las respuestas del sector privado y de las propensiones marginales a ahorrar de los gobiernos, familias y empresas. 


\section{Cuadro 2}

TASAS DE DEPENDENCIA CON LOS RESUltados (CORREgIDOS) DE LEFF PARA LOS PAÍSES QUE CONSTITUYEN PUNTOS EXTREMOS

(regresión para $\ln \mathrm{X} / \mathrm{Y}$ )

\begin{tabular}{|c|c|c|}
\hline \multirow{2}{*}{ Variables independiente } & \multicolumn{2}{|c|}{ Equación } \\
\hline & 5 & 6 \\
\hline $\ln Y / \mathbb{N}$ & $\left(\begin{array}{l}0.153^{\mathrm{b}} / \\
(1.800)\end{array}\right.$ & $\left(\begin{array}{l}0.099 \\
0.913\end{array}\right)$ \\
\hline In $D_{1}$ & $\begin{array}{c}-0.803 \\
(-1.372)\end{array}$ & $\begin{array}{c}-0.349 \\
(-0.312)\end{array}$ \\
\hline In $\mathrm{D}_{2}$ & $\begin{array}{c}-0.298 \\
(-1.311)\end{array}$ & $\begin{array}{l}-0.283 \\
(-1.131)\end{array}$ \\
\hline$\because$ & 0.119 & 0.047 \\
\hline$F$ & 1.761 & 0.529 \\
\hline Numero de palseb & $43^{c /}$ & $36^{\mathrm{d} /}$ \\
\hline
\end{tabular}

a La notación es la misma que la del cuadro 1 . Se excluyó $\ln g$ por no ser significativo (véase el texto). Todas las regresiones incluyen un término constante y positivo.

b Significativo al nivel de 0.1 .

c De los 47 originales se excluyeron los cuatro países extremos más importantes: Israel, Grecia, España y Puerto Rico.

d 36 "verdaderos países en desarrollo" igual que en $c$ excluidos además Argenitina, Chipre, Jamaica, Malta, Portugal, Trinidad y Venezuela.

ciones 7 a 9 con la ecuación 5 del cuadro 2 , se observa que nuestra ecuación produce mejores resultados. Este resultado es válido para un amplio rango tanto de formas funcionales como de selección de muestras. ${ }^{18}$ De cualquier modo, el bajo valor de $R^{2}$ sugiere la necesidad de incluir nuevas variables explicativas, a fin de obtener parámetros más confiables que los hasta ahora incluidos. La inclusión de variables adicionales, en la ecuación para $O_{m m} / Y(\mathrm{y} Y / N)$, algunas de las cuales han sido utilizadas en estudios tranšversales más heterogéneos, resultaron poco significativas para los países en desarrollo. ${ }^{19} \mathrm{Al}$ mismo tiempo que la variable

18 Ya sea que se incluyan razones no-logarítmicas $(S / Y, Y / N \ldots$, ) o bien logaritmos, se utilizaron variables simples $(\ln s, \ln Y)$.

19 Estos incluyen la tasa de inflación, importancia del sector gobierno en la economía, la "monetarización" de la economía, la densidad de población y la distribución funcional de ingreso. Se elaboraron distintas pruebas con la tasa de inflación la cual se define como el porciento de cambio en el costo de vida (o en pocos casos a trävés de los índices de precios al consumidor o de precios al mayoreo); la importancia del sector gobierno en la economía, definida como la razón entre los ingresos gubernamentales procedentes de todas las fuentes y a todos los niveles, y el producto interno bruto; el grado de monetarización de la economía, que se mide como la razón entre el circulante más la demanda de depósitos y depósitos a plazo en manos del público y el producto interno bruto; la densidad de población, definida en forma simple como el número de persona por 
clave $D_{1}$ renueva su importancia (ecuaciones 7 a 9) un análisis ulterior sugiere que esto obedece, de nueva cuenta, a la incorporación de países marginales (véanse las ecuaciones 10 y 11 ).

\section{Cuadro 3}

Modelo Alternativo de Regresión (regresión para $\ln \mathrm{S} / \mathrm{Y}$ )

\begin{tabular}{|c|c|c|c|c|c|c|c|}
\hline \multirow{2}{*}{ Bonaolsn } & \multirow{2}{*}{$\begin{array}{c}\text { Námero } \\
\text { de pafases }\end{array}$} & \multicolumn{6}{|c|}{ Variable indepondiento ${ }^{8}$} \\
\hline & & $\ln 0 \mathrm{~mm} / \mathrm{Y}$ & In $D_{1}$ & In $\mathrm{D}_{2}$ & $\ln x / x$ & $\overline{\mathrm{a}}^{2}$ & 7 \\
\hline 7 & $47^{\mathfrak{b} /}$ & $\begin{array}{c}0.319^{\circ} \\
(3.236)\end{array}$ & $\begin{array}{l}-1.351 \text { of } \\
(-2.747)\end{array}$ & $\begin{array}{l}-0.432^{\circ} / \\
(2.058)\end{array}$ & $\begin{array}{c}0.091 \\
(1.192)\end{array}$ & 0.301 & $4.515^{\circ}$ \\
\hline 8 & $43^{\underline{d}}$ & $\begin{array}{c}0.328^{\circ} \\
(3.222)\end{array}$ & $\begin{array}{l}-1.059 / \\
(-1.890)\end{array}$ & $\begin{array}{l}-0.408^{\circ} / \\
(-1.890)\end{array}$ & $\begin{array}{c}0.113 \\
(1.402)\end{array}$ & 0.261 & $3.340^{\circ}$ \\
\hline 9 & $43^{d /}$ & $\begin{array}{c}0.300^{\circ} / \\
(2.973)\end{array}$ & $\begin{array}{c}-0.868 \\
(-1.579)\end{array}$ & $\begin{array}{c}-0.322 \\
(-1.535)\end{array}$ & & 0.222 & $3.717^{-/}$ \\
\hline 10 & $36^{ \pm /}$ & $\begin{array}{c}0.254^{9 /} \\
(1.962)\end{array}$ & $\begin{array}{c}-0.819 \\
(-.734)\end{array}$ & $\begin{array}{c}-0.366 \\
(-1.513)\end{array}$ & $\begin{array}{c}0.083 \\
(0.817)\end{array}$ & 0.132 & 1.183 \\
\hline 11 & $36^{t /}$ & $\begin{array}{l}0.224^{9} \\
(1.816)\end{array}$ & $\begin{array}{l}-0.689 \\
(-.626)\end{array}$ & $\begin{array}{l}-0.320 \\
(-1.365)\end{array}$ & & 0.114 & 1.369 \\
\hline
\end{tabular}

a La notación es similar a la del cuadro 1 . Se excluyó ln $g$ por no ser significativo (véase el texto). Todas las regresiones contienen un término constante y positivo.

b Los originales 47 "países en desarrollo" de Leff.

c Significativa al nivel de 0.01 .

d De los 47 originales se excluyen los cuatro puntos extremos más importantes: Israel, Grecia, España y Puerto Rico.

- Significativa al nivel de 0.1 .

f 36 "verdaderos países en desarrollo" igual que en $d$ excluidos además Argentina, Chipre, Jamaica, Malta, Portugal y Venezuela.

Es importante subrayar que los resultados son preliminares; por lo cual no se defiende el modelo utilizado. La intención de mencionar los resultados obtenidos puede considerarse sólo como un paso adelante en el esclarecimiento de las relaciones subyacentes entre la distribución por edades y la tasa de ahorro.

hectárea de tierra cultivable; la distribución funcional del ingreso expresada como el cociente de dividir el total de sueldos y salarios entre el ingreso nacional.

Todas estas variables se examinaron tanto en el contexto de Leff y con la formulación del modelo antes citado, ninguna de estas variables resultó significativa en las regresiones. El hecho de que ninguna de las variables citadas e incluso la variable exportación, fueran significativas puede considerarse como indicación de que una alta proporción de la varianza no explicada en $S / Y$ se deba a "ruidos" (véase el apéndice A) o bien a factores no-económicos. $\mathrm{El}$ análisis de tales factores rebasa el objetivo de este trabajo. 


\section{Correcciones de los resultados de LefF para América latina}

Leff, en una comunicación posterior en esta revista [25a] presentó sus resultados estadísticos para los países latinoamericanos. Su ecuación 1 en la página 381 [25a] se reproduce aquí como la ecuación (12) en el cuadro 4 . Según Leff los resultados sugieren que:

\section{Cuadro 4}

Resultados para los países Latinoamericanos (Regresión por $\ln \mathrm{S} / \mathrm{Y}$ )

\begin{tabular}{|c|c|c|c|c|c|}
\hline \multirow{2}{*}{$\begin{array}{c}\text { Variables } \\
\text { indepordienteg }\end{array}$} & \multicolumn{5}{|c|}{ Eouao16n } \\
\hline & 12 & 13 & 14 & 15 & 15 \\
\hline $\ln Y / \mathbb{N}$ & $\begin{array}{l}0.050 \\
(0.360)\end{array}$ & $\left(\begin{array}{l}0.013 \\
(0.081)\end{array}\right)$ & $\left(\begin{array}{c}-0.307 \\
0.141)\end{array}\right)$ & & \\
\hline ln $0_{1}$ & $\left(-2.008^{b /} / 10\right)$ & $\left(\begin{array}{c}-2.570^{3 /} / \\
(1.898)\end{array}\right.$ & $\left(\begin{array}{c}-1.403 \\
(0.583)\end{array}\right.$ & $\begin{array}{c}-0.523 \\
(0.358)\end{array}$ & $\left(\begin{array}{l}1.256 \\
(0.451)\end{array}\right.$ \\
\hline In $D_{2}$ & $\left(\begin{array}{c}-0.625 \\
1.240\end{array}\right)$ & $\begin{array}{c}-1.159 \mathrm{~b} / \\
(2.034)\end{array}$ & $=\left(\begin{array}{c}-0.832 \\
(1.277)\end{array}\right.$ & $\begin{array}{c}-0.290 \\
(0.532)\end{array}$ & $\left(\begin{array}{l}0.151 \\
0.201)\end{array}\right)$ \\
\hline $\ln s$ & $\left(\begin{array}{l}0.034^{9} \\
2.050)\end{array}\right.$ & $\left(\begin{array}{l}0.147 \\
1.131)\end{array}\right.$ & $\left(\begin{array}{l}0.175 \\
1.118\end{array}\right)$ & & \\
\hline In $0 \mathrm{num} / \mathrm{I}$ & & & & $\left(\begin{array}{l}0.401 \% \\
(2.181)^{\circ}\end{array}\right.$ & $\left(\begin{array}{l}0.439^{b} \\
(1.853)\end{array}\right.$ \\
\hline $\ln x / Y$ & & & & $\left(\begin{array}{l}0.421 \\
0.038\end{array}\right)$ & $\left(\begin{array}{l}0.016 \\
0.133)\end{array}\right)$ \\
\hline $\overrightarrow{\mathrm{R}}^{2}$ & 0.271 & $\begin{array}{l}0.263 \\
1.16\end{array}$ & $\begin{array}{l}0.174 \\
0.53\end{array}$ & $\begin{array}{l}0.401 \mathrm{~b} / \\
2.18 \mathrm{~g}\end{array}$ & $\begin{array}{l}0.313 \\
1.14\end{array}$ \\
\hline Uúnero de gaíses & 18 & 18 & 15 & 18 & 15 \\
\hline
\end{tabular}

a La notación es similar a la del cuadro 1. Todas las regresiones incluyen un término constante y positivo. $R^{2}$ no está ajustada por los grados de libertad con excepción de la ecuación 12.

b Significativo al nivel de 0.1 .

c Significativo al nivel de 0.01 .

d No lo incluyó Leff.

El cociente de dependencia infantil, $D_{1}$ es un determinante significativo de las tasas de ahorro... las tasas de ahorro agregado en América Latina son reducidas por las elevadas tasas de dependencia infantil (pp. 380-381).

El primer problema que se aprecia en los resultados de Leff para América Latina es en la determinación de los 18 países a que se refiere. En su muestra incluye 20 países. De acuerdo con la discusión de la sección I, existen dos países para los cuales no se puede hacer la transforración logarítmica dado que los valores de la variable $g$ son idénticos a 
cero: República Dominicana y Uruguay. Con estos dos países excluidos de los 20 originales, los resultados se presentan en la ecuación 13. La significación estadística de $g$ desaparece pero en cambio la variable dependencia cobra relevancia. Siguiendo las mismas líneas de mi argumentación en las secciones II y III, se discute brevemente la ecuación estadística.

En primer lugar, los resultados están influidos por la selección de países, por lo cual no es posible derivar generalizaciones. Los coeficientes para $\ln D_{1}$ y $\ln D_{2}$ en la ecuación 13 (o en la 12) son significativos al nivel de $10 \%$. Si se incluyen Argentina y Venezuela, las variables pierden su utilidad estadística, como se corrobora en la ecuación 15 (se puede ver en la gráfica 1 que esto obedece a la exclusión de Argentina, que constituye un punto extremo). Por supuesto, Leff ha caído en la trampa, tan común en el análisis de los países latinoamericanos, de incluir de manera indiscriminada a los países no obstante las fuertes diferencias que hay entre algunos de ellos. En el contexto actual, dichas diferencias son económicas y demográficas, en particular en el caso de Argentina, Puerto Rico y Uruguay. Estos tres países por sus características se asemejan más a los países desarrollados que a los subdesarrollados.

Un segundo áspecto es que la estructura de la economía es más importante que el simple nivel de desarrollo, aspecto que ya se discutió en la sección III. El mejor valor explicativo de la variable $O_{m m} / Y$ se aprecia con claridad al comparar las ecuaciones 15 y 13 o las 16 y 14. Es importante destacar que la variable $O_{m m} / Y$ resulta significativa cuando se excluyen aquellos países que presentan un carácter marginal dudoso.

En resumen, Leff no demuestra la existencia de una relación negativa significativa entre las tasas de dependencia y tasas de ahorro en América Latina.

\section{AsPECTOS METODOLÓGICOS ADICIONALES}

Como es fácil observar en la gráfica 1 , basada en los datos de Leff, el rango de variación de $D_{1}$ está entre 40 y 48 , mientras que el de las tasas de ahorro, que es mayor, oscila entre 5 y $20 \%$ para países que realmente están en proceso de desarrollo.

La gráfica sugiere además que existen otros factores que son más importantes que las tasas de dependencia en la determinación de las diferencias en las tasas de ahorro entre países. Además, el bajo nivel de confiabilidad de los datos de ahorro y errores en los datos de $D_{1}$ (los que son grandes en relación con su rango de variación) sugiere que casi sería necesario adoptar un "salto de fe" kierkegaardiano para inferir relaciones de tales datos.

Aun en el supuesto de que se puedan aceptar los resultados originales de Leff y de pasar por alto las dificultades que presentan los datos, se debería de mencionar que Leff no presenta una estructura teórica que 
permita evaluar si sus coeficientes de regresión para $D_{1}$ y $D_{2}$ son o no válidos, o bien en qué dirección se da la causalidad. Por ejemplo, supóngase que un país $A$ tiene una relación $S / Y$ mayor que un país $B$, y se mantiene constante todo lo demás, en consecuencia $I / Y$ será mayor, $g$ tendería a aumentar y por lo tanto la trayectoria del ingreso del país $A$ tenderá a ser mayor a través del tiempo con respecto a la del país $B$. Dicho comportamiento estaría por lo general asociado con un mayor grado de industrialización, educación y urbanización en el caso del país $A$, el cual eventualmente alcanzaría (según la teoría de la transición demográfica) niveles más bajos de fecundidad y un $D_{1}$ más bajo. Si los resultados del análisis transversal representan en el largo plazo relaciones de "equilibrio" entre variables, la hipótesis anterior tiene la misma validez que la formulada por Leff.

Por último, se puede dudar del valor de utilizar los resultados de la regresión que se basan en un "solo disparo" a nivel transversal para luego de ahí inferir relaciones dinámicas entre las variables. (Este aspecto es evidente en Leff; véase pp. 75, 88-90 y passim).

La presencia posible de inestabilidad intraperíodo en los parámetros de la regresión sugiere que al menos deberían examinarse y compararse distintos anâlisis transversales, o más precisamente las primeras diferencias de dichas variables para luego pasar a comentar las relaciones a través de tiempo. ${ }^{20}$ y 21

\section{CONCLUSIONES}

Leff ha sugerido un nuevo enfoque para investigar empíricamente, en los países en desarrollo, las relaciones entre variables claves tanto demográficas como económicas. Pero las limitaciones de que adolece su procedimiento analítico, hacen que su artículo dé lugar a un número mayor de interrogantes que de respuestas. Las limitaciones más importantes son la ausencia de un marco teórico; el uso de un procedimiento incorrecto

20 Los parámetros estimados con base en series de tiempo y datos transversales parecen ser muy distintos: véase Kuh ([25], Cap. 6) en el caso de empresas productoras de bienes de capital en los Estados Unidos de Norteamérica en el período 1935-1955; y. Stone en relación con la demanda de los consumidores en el Reino Unido. Sus resultados sugieren de manera indirecta que no se pueden hacer inferencias directas, sin contar con evidencias de series de tiempo, que aun si los países con elevadas relaciones de dependencia ahorran menos, una disminución de las relaciones de dependencia estará asociada, ceteris paribus, con un aumento en las tasas de ahorro en el transcurso del tiempo.

21 La presente crítica se aplica también a mis resultados que aparecen en el cuadro 3. Estos se incluyen aquí como un argumento adicional para ser más cautelosos y humildes al derivar inferencias de investigaciones de tipo transversal sobre los países en desarrollo. Esto contrasta con la afirmación que hace Leff en la página 85 y con su conclusión relativa a la necesidad de políticas tendientes a reducir el crecimiento de la población, etc. Dichas políticas probablemente son deseables pero esto no se puede inferir de los resultados de Leff. 
en la medición y eliminación de la heterogeneidad de la muestra, cada uno de los cuales introduce fuertes distorsiones en los resultados empíricos, los que naturalmente apoyan su hipótesis; confiar en una hipótesis que se basa en los ahorros familiares; y en el uso de sólo un análisis transversal de los datos (débiles).

Los resultados que se desprenden de este estudio indican que la principal conclusión de Leff - que las tasas de dependencia tienen el efecto, estadísticamente significativo de deprimir las tasas de ahorro- no puede ser aceptada con la información que se utiliza.

\section{APÉNDICE A}

\section{DESCRIPCIÓN DE LOS DATOS}

Fuentes de información. Los países estudiados aparecen en el artículo de Leff [25], quien gentilmente me cedió la información sobre $S / Y, S / N, Y / N$, $g, D_{1}$ y $D_{2}$. Las fuentes de los datos restantes que se utilizaron son: $O_{m m}$, Naciones Unidas [38], 1968 y OECD [30], excepto para Ghana, las cuales se obtuvieron de W. D. Reddaway [32], p. 107. Los datos de importaciones y exportaciones, el índice del costo de vida, ingresos agregados del gobierno, y cuasi-dinero (véase la nota 20 en el texto), Fondo Monetario Internacional [16].

Los de tamaño de la población en 1964, Naciones Unidas [35], 1968. Los de sueldos, salarios e ingreso nacional, Naciones Unidas [38].

La información sobre tierra cultivable, Naciones Unidas, Organización para la Alimentación y Agricultura [39].

Calidad de los datos. Es de sobra conocido que entre los datos macroeconómicos, los que se refieren al ahorro son los menos confiables en los países en desarrollo (debido a que los ahorros en las cuentas nacionales normalmente se calculan en forma residual) y que las cifras sobre ingreso per capita en rigor no son comparables entre países; por lo que Leff debería haber utilizado el producto nacional bruto en lugar del producto interno bruto a fin de hacer comparaciones transversales entre países (Véase Abraham [1], p. 379, y Landau [23], p. 165).

Los datos de población adolecen también de errores, los que si bien son más pequeños respecto a sus verdaderos valores, tienen importancia para la clase de estudio elaborado por Leff, en el cual los verdaderos países de escaso desarrollo tienen valores que oscilan entre el 40 y el 48 (véase la gráfica 1) para $D_{1}$ y de 2 a 5 en el caso de $D_{2}$. Un error de 3 a $4 \%$ en el valor de $D_{1}$ puede modificar por completo la posición relativa de un país. Más aún, tales errores no son similares en los países en desarrollo de las distintas regiones [36]. De hecho, de los 47 países en desarrollo que estudió Leff, sólo 10 (la mitad de ellos son islas pequeñas), de acuerdo con las Naciones Unidas, disponían en 1964 de datos confiables sobre la composición por edades [35].

Leff introduce además una fuente adicional de error en las cifras sobre $D_{1}$ al utilizar los datos que pudo obtener de los censos para estimar las tasas de dependencia, los que maneja sin el ajuste necesario para hacerlos consistentes con el año de 1964, año a que se refieren los datos económiccs. 
Por ejemplo, algunas de las cifras que utiliza para $D_{1}$ (y $D_{2}$ ) en 1964, corresponden a 1957 (para Irak y Tanganika). Con base en la teoría de las poblaciones estables [6 y 7], los cálculos que hice, con base en los censos de postguerra para los países en desarrollo, se puede demostrar que $D_{1}$ puede aumentar $5 \%$ o más en un período de siete años en aquellos países que han registrado tasas de crecimiento entre el 2 y $3.5 \%$ (la mayoría de los países en desarrollo). Dicha fuente adicional de error (que podría evitarse) en los datos de la variable independiente $D_{1}$ aumenta los errores de medición en las otras variables, introduciendo así un elemento adicional que afecta innecesariamente las estimaciones de los parámetros de la regresión.

\section{APÉNDICE B}

\section{$S / Y$ VERSUS $S / N$ COMO VARIABLES DEPENDIENTES}

Además del poco interés teórico -es decir, países con más altos ingresos per capita tienen más altos ahorros per capita - hay dos razones de naturaleza metodológica para rechazar $S / N$ como variable dependiente: a) Las regresiones de $S / N$ sobre $Y / N$ (variable definida en el texto) entre paises depende de la comparabilidad de las estimaciones del ingreso per capita en dólares (que con frecuencia se ha mencionado en la discusión); y b) En los resultados de Leff existen correlaciones espurias para $S / N$, que explican la diferencia en el poder explicativo que tienen las variables independientes en las regresiones para $S / N$ y $S / Y$ del artículo de Leff (ecuaciones de las pp. $81-83,86$ ).

Las correlaciones entre $S / N$ y $Y / N$ son, con mucho, más altas que las de $S / Y$ y $Y / N$. Es decir, al dividir la variable $S$ y $Y$ por $N$ se crean variables que están correlacionadas de manera artificial en la medida que la variancia de $N$ se asemeja a las variancias de $S$ y $Y$. Específicamente Kuh y Meyer ([22], p. 405) demostraron que el problema de correlación espuria, producto del empleo de variables en forma de razones, surge cuando se dan en cualquiera de las siguientes condiciones: 1) El coeficiente de variación del denominador es mayor comparado con el del numerador; 2) Las variables del numerador no son funciones lineales homogéneas del denominador. Para la muestra de países en desarrollo (43 o bien los 47) existen correlaciones espurias: los coeficientes de variación para los 47 paises en desarrollo son $1.660,1.708$ y 2.977 para $S, Y$ y $N$, respectivamente. Regresiones simples de $S$ sobre $N$ y $Y$ sobre $N$ dan términos constantes diferentes de cero que son significativos al nivel del $1 \%$, lo cual indica ausencia de homogeneidad lineal. En el caso de la muestra de 70 países, los resultados indican la existencia de correlaciones espurias pero en menor grado.

Con base en lo anterior existen razones tanto de orden estadístico como metodológico que obligan a seleccionar a $S / Y$ en lugar de $S / N$ en aquellas regresiones en las que $Y / N$ es una de las variables independientes.

Cálculos similares se realizaron para hacer la prueba de correlación espuria en el modelo alternativo que se propone, ya que puede darse el caso de que haya correlación espuria en la regresión de las razones $S / Y$ y $O_{m m} / Y$. Los coeficientes de variación para $S, O_{m m}$ y $Y$ son de $1.660,1.685$ y 1.708 respectivamente (47 países). Las regresiones simples de $S$ sobre $Y$ y $O_{m n}$ so- 
bre $Y$ dan un término constante diferente de cero, que es no significativo (con todos los errores estándar al menos 10 veces el tamaño del término constante). Así, mientras este tipo de correlación espuria existe en las regresiones de $S / Y$ sobre $O_{m m} / Y$, ésta no resulta tan problemática como en las regresiones de $S / N$ sobre $Y / N$.

\section{REFERENCIAS BIBLIOGRAFICAS}

1. W. Abraham, "Saving Patterns in Latin America", Economic Development and Cultural Change, Vol. XII (4), julio de 1964, pp. 377-391.

2. I. Adelman, "An Econometric Analysis of Population Growth", American Economic Review, Vol. 53, junio de 1963, pp. 314-339.

3. N. Adams, "Dependency Rates and Savings Rates: Comment", American Economic Review, Vol. 61, junio de 1971, pp. 472-475.

4. R. Barlow y G. Davis, "Project Evaluation with a Detailed Macroeconomic Model", ms., Department of Economics, University of Michigan, 1971.

4a. H. Chenery y P. Eckstein, "Development Alternatives for Latin America", Journal of Political Economy, Vol. 78 (4), julio/agosto de 1970, Parte II, pp. 966-1006.

5. G. Chow, "Tests of Equality Between Sets of Coefficients in Two Linear Regressions", Econometrica, Vol. 28, julio de 1960, pp. 591-605.

6. A. Coale, The Growth and Structure of Human Populations: A Mathematical Investigation, Princeton, 1972.

7. A. Coale y P. Demeny, Regional Model Life Tables and Stable Populations, Princeton, 1966.

8. W. Dixon y F. Massey, Introduction to Statistical Analysis, Nueva York, 1957.

9. C. Drakatos, "The Determinants of Birth Rates in Developing Countries: An Econometric Study of Greece", Economic Development and Cultural Change, Vol. 17, Núm. 4, julio de 1969, pp. 596-603.

10. M. Friedman, A Theory of the Consumption Function, Princeton, 1957.

11. F. Grubbs, "Sample Criteria for Testing Outlying Observations", Annals of Mathematical Statistics, Vol. 21 (2), 1950, pp. 27-56.

12. K. L. Gupta, "Dependency Rates and Savings Rates: Comment", American Economic Review, Vol. 61, junio de 1971, pp. 469-471.

13. F. Harbison, J. Maruhnic, y J. Resnick, Quantitative Analysis of Modernization and Development, Industrial Relations Section, Princeton University, 1970.

14. E. Hoover, "Basic Approaches to the Study of Demographic Aspects of Economic Development: Economic-Demographic Models", Population Index, Vol. 37 (2), abril-junio de 1971, pp. 66-75.

15. H. S. Houthakker, "On Some Determinants of Savings in the Developed and Underdeveloped Countries", en E. A. G. Robinson (Comp.), Problems of Economic Development, Londres, 1965, pp. 212-226.

16. International Monetary Fund, International Financial Statistics.

17. A. Kelley, "Demand Patterns, Demographic Change, and Economic Growth", Quarterly Journal of Economics, Vol. LXXXIII (1), febrero de 1969, pp. 110-126.

18. T. King, "Private Savings", Banco Internacional de Reconstrucción y Desarrollo (manuscrito), marzo de 1970.

19. L. Klein, A Textbook of Econometrics, Evanston, 1953.

20. K. Krishnamurty, "Economic Development and Population Growth in Low- 
Income Countries: An Empirical Study for India", Economic Development and Cultural Change, Vol. 15, octubre de 1966, pp. 70-75.

21. E. Kuh, Capital Stock Growth: A Micro-Econometric Approach, Amsterdam, 1963.

22. E. Kuh y I. R. Meyer, "Correlation and Regression Estimates' when the Data are Ratios", Econometrica, Vol. 23, 1955, pp. 400-416.

23. L. Landau, "Differences in Saving Ratios among Latin American Countries", Tesis doctoral, Harvard University, junio de 1969.

24. J. Lee, "Exports and the Propensity to Save in L.D.C.s", Economic Journal, Vol. 81 (322), junio de 1971.

25. N. Leff, "Dependency Rates and Savings Rates", American Economic Review, Vol. 59, Núm. 5, diciembre de 1969, pp. 886-896; en español: "Tasas de natalidad y tasas de ahorro", Demografía Y Economía, Vol. III (1), 1969, pp. 75-93.

25a. "Tasas de dependencia y tasas de ahorro en América Latina", Demografía y ECoNomí, Vol. IV (3), 1970, pp. 379-381.

26. N. Leff, "Dependency Rates and Savings Rates: Reply", American Economic Review, Vol. 61, junio de 1971, pp. 476-480.

27. B. F. Massell y J. Heyer, "Household Expenditure in Nairobi: A Statistical Analysis of Consumer Behavior", Economic Development and Cultural Change, Vol. 17, Núm. 2, enero de 1971, pp. 476-480.

28. D. McGranahan, "Analysis of Socio-Economic Development Through a System of Indicators", The Annals of the American Academy of Political and Social Science, enero de 1971, pp. 65-81.

29. F. Modigliani, "The Life Cycle Hypothesis of Saving and Intercountry Differences in the Saving Ratio", en W. Eltis (Comp.), Induction, Growth and Trade, Oxford, 1970, pp. 197-224.

30. Organization for Economic Cooperation and Development, National ACcounts of Less Developed Countries, París, 1969.

31. L. Phillips, H. Votey y D. Maxwell, "A Synthesis of the Economic and Demographic Models of Fertility: An Econometric Test", Review of Economics and Statistics, Vol. 51, Núm. 3, 1969, pp. 298-308.

32. W. D. Reddaway, "The Future of the Ghanian Economy", en I. D. Stewart (Comp.), Economic Development and Cultural Change, Edinburgo, 1969, p. 107.

33. W. Robinson y D. Horlacher, "Population Growth and Economics Welfare", Reports on Population/Family Planning, Núm. 6, The Population Council, junio de 1971.

34. J. Stone, The Measurement of Consumers Expenditure and Behavior in the United Kingdom, 1920-1938, Cambridge, 1954.

35. Naciones Unidas, Demographic Yearbook, Varios números.

36. Naciones Unidas, Manual IV: Methods of Estimating Basic Demographic Measures from Incomplete Data, Population Studies, Núm. 42, Nueva York, 1967.

37. Naciones Unidas, World Economic Survey, 1969-70, Nueva York, 1971.

38. Naciones Unidas, Yearbook of National Accounts Statistics, 1968, Nueva York, 1969.

39. Naciones Unidas, Food and Agricultural Organization, Production Yearbook, 1966. 